ORIGINAL ARTICLE

\title{
Non-clinical factors influencing the selection of patients with acute coronary syndromes for angiography
}

\author{
A Quaas, N Curzen, C Garratt
}

Postgrad Med J 2004;80:411-414. doi: 10.1136/pgmi.2003.011247

See end of article for authors' affiliations

......................

Correspondence to: Dr Alexander Quaas, c/o Manchester Heart Centre, Manchester Royal Infirmary, Oxford Road Manchester M139WL, UK; alex_quaas@hotmail.com

Submitted 13 June 2003 Accepted 29 August 2003
Objective: To analyse clinical and non-clinical factors determining the selection for coronary angiography in patients with acute coronary syndromes (ACS).

Design: Single centre, prospective cohort study.

Participants: Eighty consecutive patients admitted with a diagnosis of ACS during the period 21 May 2001 to 4 July 2001.

Setting: Coronary care unit of a tertiary referral centre, the Manchester Royal Infirmary.

Data collection: Information concerning baseline patient characteristics, clinical presentation, and the selection for angiography was collected from the patient notes.

Data analysis: Windows SPSS version 9.0 using cross tabulations with $\chi^{2}$ estimation and binomial logistic regression analysis.

Main outcome measure: Selection for angiography in ACS.

Results: Cross tabulations with $\chi^{2}$ analysis and logistic regression analysis identified significant nonclinical factors predicting the use of angiography. Although clinical factors such as recurrent ischaemia (odds ratio 5.11) influenced the decision to undergo coronary angiography, non-clinical factors such as young age (odds ratio 6.88 for $<65$ years old), gender (odds ratio 3.81 for males), admission on a weekday (odds ratio 0.2488 for admission on the weekend), and consultant in charge (odds ratio 0.111 for consultant " 2 ") independently predicted the use of angiography in ACS.

Conclusion: The selection of patients for angiography in ACS is not based purely on clinical criteria. Awareness of the apparent sources of bias among clinical decision makers may improve management of these patients.
Numerous clinical and non-clinical variables for each patient included in this study were used to "screen" for potential predictive factors for the use of coronary angiography in ACS.

\section{METHODS}

\section{Patient selection}

The studied cohort consisted of 80 consecutive patients admitted to the coronary care unit of Manchester Royal Infirmary University Hospital with a diagnosis of ACS during the period from 21 May 2001 until 4 July 2001.

The inclusion criteria were coronary care unit admission during the above period and the presence of one of the ACS. ACS were defined as a constellation of a consistent history of cardiac chest pain and laboratory results suggestive of either non-ST elevation myocardial infarction (NSTEMI) or unstable angina. Classification was based upon ischaemic changes on the electrocardiogram ( $\mathrm{T}$ wave inversion or ST segment depression) and/or raised troponin $\mathrm{T}$ concentrations.

All patients were admitted as emergencies to the cardiology consultant on-call, and the pattern of referral was the same for all consultants.

Patients admitted to the coronary care unit with conditions unrelated to ACS-for example, cardiac arrhythmias-were excluded from the study.

Data collection was conducted from case notes. artery bypass grafting (class I); patients with "repeated presentations for ACS"; and "patients over 65 years old" (class IIa).

In this study, we analysed whether the selection of ACS patients for angiography is dependent purely upon the above clinical criteria and, if not, which non-clinical factors determine the selection in clinical reality.

\section{Data analysis}

Statistical analysis was performed using SPSS version 9.

Abbreviations: ACS, acute coronary syndromes; NSTEMI, non-ST elevation myocardial infarction; OR, odds ratio 
Cross tabulations with $\chi^{2}$ estimations were used to analyse correlations between the presence or absence of certain observed patient characteristics and the subsequent use of coronary angiography. The null hypothesis that the analysed variable and the selection for angiography are independent was tested.

Logistic regression analysis with specified independent variables was used to predict the binary outcome "in-hospital use of coronary angiography". Odds ratios were calculated, estimating by which factor the ratio between the complementary outcomes "angiography" and "no angiography" increased when the independent variable was increased by one unit.

In all analyses, $\mathrm{p}<0.05$ was considered statistically significant.

\section{RESULTS}

Overall, 48 patients out of the 80 in the study population were selected for coronary arteriography. This represented $60 \%$ of the total number of patients. Two subgroups were created based on this dichotomous outcome.

The characteristics of the cohort are given in table 1 .

Forty five percent of patients were older than $65,65 \%$ were male, $72.5 \%$ were current or previous smokers, $52.5 \%$ were diagnosed hypertensive, $57.5 \%$ diagnosed hypercholesterolaemic, almost a fifth $(18.8 \%)$ of patients were diabetic (all type II diabetes mellitus), and nearly half the patient population $(46.3 \%)$ had at least one first degree relative with ischaemic heart disease. For exactly $50 \%$ of patients it was

\begin{tabular}{|c|c|}
\hline Variable & $\begin{array}{l}\text { No (\%) of } \\
\text { patients }\end{array}$ \\
\hline \multicolumn{2}{|l|}{ Age and gender } \\
\hline Mean age (years) & 61.4 \\
\hline Age $<65$ & $44(55)$ \\
\hline Female & $28(35)$ \\
\hline \multicolumn{2}{|l|}{ Mode of admission } \\
\hline Acute medical admission & $59(73.8)$ \\
\hline $\begin{array}{l}\text { Urgent transfer from district general } \\
\text { hospital }\end{array}$ & $21(26.3)$ \\
\hline \multicolumn{2}{|l|}{ Coronary risk factors } \\
\hline Smoker & $58(72.5)$ \\
\hline Hypertensive & $42(52.5)$ \\
\hline Raised cholesterol (>5.0 mmol/l) & $46(57.5)$ \\
\hline Positive family history & 37 (46.3) \\
\hline Diabetes & $15(18.8)$ \\
\hline Positive cardiac history & $40(50)$ \\
\hline \multicolumn{2}{|l|}{ Previous cardiac disease } \\
\hline Previous myocardial infarction & $24(30)$ \\
\hline Previous angina & $32(40)$ \\
\hline Previous CABG & $7(8.8)$ \\
\hline Previous PCl & $17(21.3)$ \\
\hline \multicolumn{2}{|c|}{ Non-cardiac comorbidity } \\
\hline $\begin{array}{l}\text { Significant medical history (for example, } \\
\text { previous CVA, asthma) }\end{array}$ & $44(55)$ \\
\hline \multicolumn{2}{|l|}{ Presentation } \\
\hline Chest pain at rest & $80(100)$ \\
\hline Abnormal ECG & $59(73.8)$ \\
\hline ST depression & $31(38.8)$ \\
\hline T wave inversion & $36(45)$ \\
\hline Raised troponin T concentration & $56(70)$ \\
\hline \multicolumn{2}{|l|}{ Type of ACS } \\
\hline Unstable angina & $24(30)$ \\
\hline NSTEMI & $56(70)$ \\
\hline \multicolumn{2}{|l|}{ Risk stratification } \\
\hline "High risk" (BCS) & $56(70)$ \\
\hline Recurrent ischaemia & $41(51.3)$ \\
\hline
\end{tabular}

the first presentation of cardiac disease, whereas the other half were "known" ischaemic heart disease patients, $42.6 \%$ of whom had had angiographic investigation of their coronary arteries in the past.

Fifty nine of the 80 patients were acute medical admissions whereas 21 were emergency transfers from district general hospitals. Chest pain at rest was the main presenting complaint in all patients.

The electrocardiogram was interpreted as abnormal in $73.8 \%$ of patients. ST segment depression occurred in $38.8 \%$ of patients, and abnormal $\mathrm{T}$ wave inversion in $45 \%$, either isolated or in combination.

Seventy percent of the population had evidence of myocardial injury as measured by troponin $\mathrm{T}$ concentrations in the systemic circulation. These patients fell into the subgroup NSTEMI, the remainder $(30 \%)$ were diagnosed with unstable angina.

The troponin $\mathrm{T}$ positive patients also represented the "high risk" group according to the British Cardiac Society. Altogether $51.3 \%$ of patients had ongoing chest pain during their admission after initial management. This phenomenon was recorded as "recurrent ischaemia".

To analyse the role of clinical and non-clinical factors in the selection for coronary angiography in patients with ACS, an initial "screening" of variables using cross tabulations and $\chi^{2}$ testing was performed.

Variables were recorded for which the null hypothesis of independence of angiography selection had to be rejected, meaning that they are significantly associated with the use of the technique.

Identified significantly associated clinical factors included "recurrent ischaemia" (Pearson's $\chi^{2}=9.925 ; p=0.002$ ) and "British Cardiac Society risk class" (Pearson's $\chi^{2}=6.949$; $\mathrm{p}=0.031$ ). Of the 56 "high risk" patients with positive troponin concentrations (70\% of the sample), 38 patients $(67.9 \%)$, underwent angiography. Of the 24 patients who were negative for troponin, $10(41.6 \%)$ received the investigation.

A significant association with the use of angiography was found for the non-clinical factors "age over 65" (Pearson's $\left.\chi^{2}=13.807 ; \mathrm{p}<0.001\right)$, "gender" $\left(\right.$ Pearson's $\chi^{2}=6.432$; $\mathrm{p}=0.011)$, "consultant in charge" (Pearson's $\chi^{2}=13.134$, $\mathrm{p}=0.011$ ), and "weekday of admission" (Pearson's $\left.\chi^{2}=15.918, p=0.014\right)$. For the latter variable, differences in the rates of angiography use were observed, ranging from $89 \%$ on a Tuesday to $20 \%$ on a Sunday (fig 1 ).

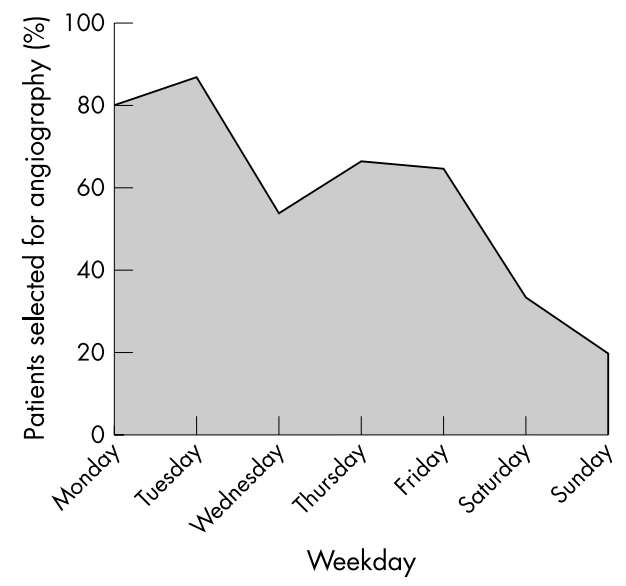

Figure 1 Angiography rates according to weekday of admission (percentage of patients undergoing angiography of patients admitted on that weekday). 


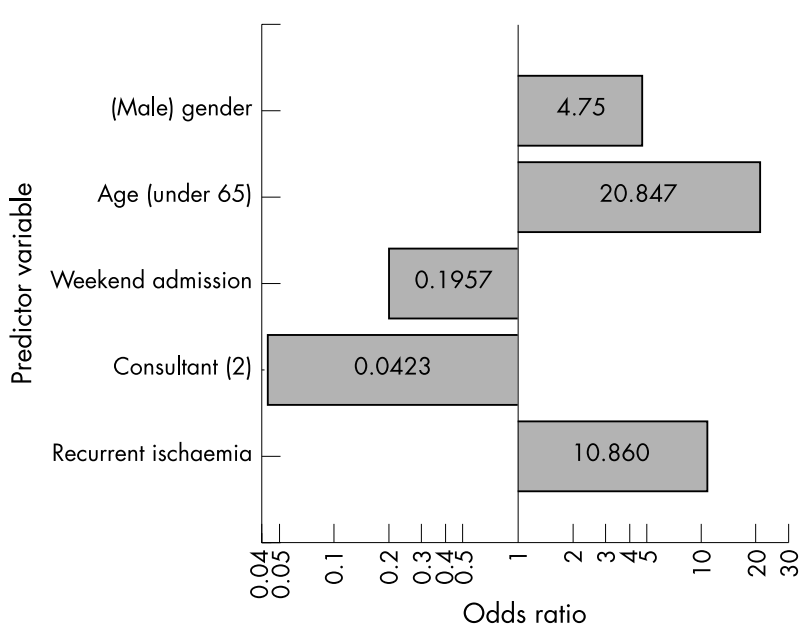

Figure 2 Odds ratios for the independent predictor variables included in the model. Note that ratios are represented on a logarithmic scale with reference to an odds ratio $=1$. The variables "weekend admission" $(O R=0.1957 ; p=0.0053)$ and "consultant (2) in charge"

$(O R=0.0423 ; p=0.0298)$ had odds ratios $<1$ and therefore negatively predicted the use of angiography. The variables "male gender"

$(O R=4.7505 ; p=0.0302)$, "age under 65" $(O R=20.847 ; p=0.011)$, and recurrent ischaemia $(O R=10.860 ; p=0.0043)$ had odds ratios $>1$; they were positive independent predictors of the use of angiography.

Logistic regression analysis was used to predict the dichotomous outcome variable "angiography selection" from a set of independent regressors.

In a preliminary "screening process", variables were tested for their independent contribution to a logistic regression model predicting the use of angiography. This was done estimating the difference in -2 log likelihood between a model with and without the variable tested.

Variables identified with predictive potential were included in a logistic regression model. These were the clinical factor "recurrent ischaemia" and the non-clinical factors "gender", "age under 65", "weekend admission", and "consultant (2) in charge".

The $\chi^{2}$ value of the model was $52.586(\mathrm{p}<0.001)$, indicating that the "global null hypothesis" can be reliably rejected.

All of the variables included independently and significantly contributed to the model. The odds ratios (ORs) obtained are the factors by which the ratio of the two complementary outcomes changes when the independent variable is increased by one unit (fig 2).

The variable "recurrent ischaemia" was the only analysed clinical factor that had a big enough individual contribution to a potential logistic-regression model to be included (OR 10.860; $\mathrm{p}=0.0043$ ).

All of the tested non-clinical variables "male gender" $(\mathrm{OR}=4.7505 ; \mathrm{p}=0.0302)$, “age under 65" $(\mathrm{OR}=20.847$; $\mathrm{p}=0.011)$, "weekday group" $(\mathrm{OR}=0.1957 ; \mathrm{p}=0.0053)$, and "consultant (2) in charge" (OR $=0.0423 ; \mathrm{p}=0.0298)$ were independent predictors of the use of angiography. Being male and aged under 65 were positively predictive (OR $>1$ ) whereas admission on the weekend and under consultant (2) were negatively predictive $(\mathrm{OR}>1)$ of the binary outcome variable "selection for angiography".

\section{DISCUSSION \\ Clinical and non-clinical factors in the selection for coronary angiography in ACS}

In this study, the selection of ACS patients for angiography is not purely based on evidence based clinical criteria. Although established criteria such as troponin $\mathrm{T}$ concentrations and recurrent ischaemia influence the selection, non-clinical factors have an important role.

The most important clinical element influencing the use of angiography was recurrent ischaemia. Only nine out of 41 patients with recurrent ischaemia were not selected, whereas this was the case for 23 out of the 39 without further ischaemic symptoms after admission. The variable was the only analysed clinical factor that had a big enough individual contribution to a potential logistic regression model to be included.

In the model, it increased the odds of being selected for angiography independently by a factor of $10.86(p=0.0043)$. Recurrent chest pain, often referred to as "failed reperfusion", seems to guide clinicians heavily towards an invasive strategy.

No independent predictive effect, but a significant association with the use of angiography, was found for the British Cardiac Society risk class. This classification is based on troponin $\mathrm{T}$ concentrations and the result of non-invasive stress testing. The latter was not performed in the acute setting, so the 56 patients with positive troponin concentrations (70\% of the sample) were "high risk" according to the British Cardiac Society. Of these, a majority (67.9\%), but not all patients underwent angiography; $41.6 \%$ of the troponin negative patients received the investigation. No effect of the variable "previous angiography" on the selection for angiography on this admission was noted.

Regarding non-clinical factors, the variables "male gender" and "age under 65 " were positive independent predictors of the use of angiography, the variables "weekend admission" and "consultant (2) in charge" negatively predicted the use of angiography. No independent effect of the mode of admission was found.

By far the most influential predictor for the use of angiography was young age. The odds ratio implies that merely being younger than 65 years old increased the odds of undergoing angiography by a factor of more than 20, independent of any other variable. Notably, this phenomenon was independent of factor "comorbidity" in the logistic regression model. Therefore the observed bias against elderly patients cannot be explained by concurrent significant noncardiac disease. This strategy is inconsistent with current guidelines ${ }^{3}{ }^{4}$ and may introduce bias against elderly patients at high risk, who would be the most likely to benefit.

This topic has previously been discussed in various settings. Pilote et al evaluated determinants of the use of coronary angiography after thrombolysis for acute myocardial infarction with various regression models. ${ }^{5}$ They found that "younger age and the availability of the procedures appeared to be the major determinants of the use of coronary angiography" suggesting that "this process appeared to select lowrisk patients for intervention rather than those at higher risk, who would be the most likely to benefit".

This phenomenon may occur as a consequence of physician preference. ${ }^{6}$ When cardiologists in Scotland were surveyed by postal questionnaire asking them to detail their approach to four sample clinical scenarios, substantial differences in practice were observed in the management of subjects with non-Q wave myocardial infarction. Of the cardiologists surveyed, $40 \%$ would undertake coronary angiography irrespective of the results of non-invasive testing in a 45 year old patient, but only one would adopt the same policy in an otherwise fit 77 year old. Only 44\% would perform any investigations (beyond echocardiography) in the 77 year old.

A similar bias, proved in this study, concerns the variable "gender". Male gender was an independent predictor for the use of angiography. Many studies have shown in the past that women are less likely than men to be referred for coronary angiography. ${ }^{7-9}$ Often, gender and age were 
discussed together because of their similar effect. A recent retrospective analysis of patients' medical case notes $(n=1790)$ from a single UK district hospital in the Thames Region ${ }^{10}$ suggested that "the management and treatment of older patients and women with cardiac disease may be different from that of younger patients and men. Given the similarity of the indications for treatment and the lack of significant contraindications or comorbidities as a cause for these differences, one possible explanation is that these patients are being discriminated against principally because of their age and sex".

In this study, the factors age and gender both had independent effects in the logistic regression model. Therefore, the lower angiography rates for females cannot be explained by the fact that they were older as well (and vice versa).

An interesting result is the identification of the variable "weekend admission" as an independent predictor for the use of angiography. The variable "day of admission" was not necessarily the day of the procedure. For example, if a patient was admitted on Saturday and underwent angiography the next Tuesday, this was noted as "angiography done" for a "Saturday admission". However, in most cases, angiography was performed within 24 hours. Lower angiography rates towards the end of the week were observed (fig 1). A likely explanation for this may be that less staffed catheter laboratories only open for selected emergency cases on the weekend days despite continuing admission and transfer of patients with ACS. In a similar fashion, variations in the availability of the catheter laboratory between district general hospitals and tertiary referral centres are known to influence waiting times for angiography. ${ }^{11}$ In this patient sample, interhospital transfers represented a minority of cases ( 21 cases) that were admitted "with a view to coronary angiography". The consultant on-call was in charge of the decision whether to accept the transfer or not, and the decision whether to go ahead with angiography after the transfer had occurred.

A high impact of the cardiologist in charge was noted. Although it has been advocated that an aggressive approach is indicated in all "high risk" (that is, troponin positive) patients with $\mathrm{ACS}^{2{ }^{3}}$ there is a marked discrepancy among clinicians in adopting this approach. We suspect that this accounts for the fact that the troponin concentration was not an independent predictor of angiography selection. The lack of conformity of clinical practice to the established evidence base exposes patients to heterogeneity of clinical standards and is unacceptable.

\section{Conclusion}

Our results suggest that, in making decisions which patients with ACS to refer for angiography and revascularisation, doctors may be influenced by non-clinical factors unrelated to established criteria for detection of risk and proof of benefit.

Rigorous audit tools, applied widely and frequently, may reduce such heterogeneous clinical practice and variable standards of care.

\section{Authors' affiliations}

A Quaas, N Curzen, C Garratt, Manchester Heart Centre, Manchester Royal Infirmary, UK

\section{REFERENCES}

1 Murray CJL, Lopez AD. Mortality by cause for eight regions of the world: global burden of disease study. Lancet 1997;349:1269-76.

2 Smith E, Fath-Ordoubadi F, Curzen N. Unstable angina: the case for selective aggression. Br J Cardiol 2002;9:251-4.

3 Anonymous. Guideline for the management of patients with acute coronary syndromes without persistent ECG ST segment elevation. British Cardiac Society Guidelines and Medical Practice Committee and Royal College of Physicians Clinical Effectiveness and Evaluation Unit. Heart (British Cardiac Society: Online) 2001;85:133-42.

4 New Guidelines for Evaluating Acute Coronary Syndrome. The American College of Cardiology and the American Heart Association. Clinician Reviews 2001;11:73-86.

5 Pilote L, Miller DP, Califf RM, et al. Determinants of the use of coronary angiography and revascularization after thrombolysis for acute myocardia infarction. N Engl J Med 1996;335:1 198-205.

6 Mahy IR, Hillis GS, Jennings KP. Risk stratification after myocardial infarction in Scotland. Scott Med J 1998;43:72-3.

7 Ayanian JZ, Epstein AM. Differences in the use of procedures between women and men hospitalised for coronary disease. N Engl J Med 1991;325:221-5.

8 Kromholz H, Douglas P, Laver M, et al. Selection of patients for coronary angiography and coronary revascularisation after myocardial infarction: is there evidence for a gender bias? Ann Intern Med 1992;166:785-90.

9 Heller LI. Diagnostic evaluation of women with suspected coronary artery disease. Cardiology 1995;86:318-23.

10 Bowling A, Bond M, McKee D, et al. Equity in access to exercise tolerance testing, coronary angiography, and coronary artery bypass grafting by age, sex and clinical indications. Heart 2001;85:680-6.

11 Miller C, Lipscomb K, Curzen N. Are district general hospital patients with unstable angina at a disadvantage? Postgrad Med J 2003;79:93-8. 